USACH/99/01，DFTUZ/99-02

\title{
Light-cone quantization of two dimensional field theory in the path integral approach
}

\author{
J.L. Cortés ${ }^{1 *}$ and J. Gamboa ${ }^{2 \dagger}$, \\ ${ }^{1}$ Departamento de Física Teórica, Universidad de Zaragoza, Zaragoza 50009, Spain \\ ${ }^{2}$ Departamento de Física, Universidad de Santiago de Chile, Casilla 30\%, Santiago 2, Chile
}

\begin{abstract}
A quantization condition due to the boundary conditions and the compatification of the light cone space-time coordinate $x^{-}$is identified at the level of the classical equations for the right-handed fermionic field in two dimensions. A detailed analysis of the implications of the implementation of this quantization condition at the quantum level is presented. In the case of the Thirring model one has selection rules on the excitations as a function of the coupling and in the case of the Schwinger model a double integer structure of the vacuum is derived in the light-cone frame. Two different quantized chiral Schwinger models are found, one of them without a $\theta$-vacuum structure. A generalization of the quantization condition to theories with several fermionic fields and to higher dimensions is presented.
\end{abstract}

Typeset using REVTEX

*E-mail: cortes@leo.unizar.es

†E-mail: jgamboa@lauca.usach.cl 


\section{INTRODUCTION}

Light-cone quantization (LCQ) has been considered recently as an appropriate framework for a non-perturbative study of relativistic quantum field theory [1 [3]. The structure of the vacuum and bound states are examples of ingredients which have a very different description in LCQ compared with canonical equal-time quantization. This opens the possibility to get a new perspective on basic problems like the mechanism of confinement in non-abelian gauge theories by the use of different quantization schemes.

From a technical point of view a property of the quantization in the light cone which plays a crucial role in the present work is that it is naturally defined over a manifold with nontrivial topology. This can easily be understood from the form of the dispersion relation in the light-cone $k_{+} k_{-}-k_{\perp}^{2}=m^{2}$. In order to be able to get the light-cone energy $k_{-}$from this expression one considers discrete light-cone quantization (DLCQ) [4], i.e., a compactified $x^{-}$ coordinate, $-L \leq x^{-} \leq L$, leading to discrete non-zero values for the light-cone longitudinal momentum $k_{+}$.

The aim of this paper is to explore physical effects whose explanation is related to this implicit difference in topology from standard spacetime quantization. We concentrate the discussion on the simplest system, two-dimensional field theory, and as a tool the path integral formulation, which has proved to be very useful at least at a formal level in the derivation of the fundamental aspects of quantum field theory, is used. In the next section we consider quantum mechanics as a toy model to illustrate the general framework of the discussion. In section 3 we study the Thirring model; following the standar representation of the fermion self-coupling by an auxiliary vector field we find a quantization condition on the integral of the vector field along a constant $x^{-}$closed line. When the relation between the auxiliary field and the fermion current is taken into account and one considers a coupling $g^{2}$ such that $g^{2} / \pi$ is a rational number then the quantization condition translates into a restriction on the possible values of the number of left-handed fermions, a result obtained previously [5] by other means. In section 4 similar arguments are applied to the Schwinger 
model and the same quantization condition of the Thirring model is obtained with the auxiliary field replaced by the gauge field. In this case there is no algebraic relation between the vector field and the fermion density; as a consequence of the quantization condition on the integral involving the $A_{-}$component one finds a restriction on the asymptotic behavior at $x^{+}$equal to plus or minus infinity for the zero mode ( $x^{-}$independent component) of the scalar field whose derivative with respect to $x^{+}$gives the $A_{+}$component. This asymptotic behavior is given in terms of a second integer leading to a double integer structure for the model. We end up in section 5 with a brief discussion of possible generalizations including the case with more than one fermionic field where the quantization condition appears as an equation por the $x^{-}$path ordered exponential of the integral of an appropriate non-abelian $A_{-}$field over the constant $x^{-}$closed line.

\section{A QUANTUM MECHANICAL EXAMPLE}

In [5] we showed that for the Thirring model in the light cone, the composite $\int_{-L}^{L} d x^{-} \psi_{L}^{\dagger} \psi_{L}$ is quantized and we argued also that this quantization is due to $S^{1} \times \Re$ topology induced by the light cone frame. In this section we will show that this quantization rule is quite natural and it is always present in the quantization of physical systems when periodic or antiperiodic boundary conditions are assumed.

We illustrate our argument considering the quantum mechanics of a particle with an action [6]

$$
S=\int_{0}^{T} d t\left[\frac{\dot{x}^{2}}{2}-V-\psi^{\dagger}\left(i \partial_{t}+W\right) \psi\right]
$$

where $V=V(x), W=W(x)$ and $x$ and $\psi$ are bosonic and fermionic degrees of freedom respectively. In order to compute $Z$ we must fix the boundary conditions as follows

$$
\begin{aligned}
& x(0)=x(T), \\
& \psi(0)=-\psi(T),
\end{aligned}
$$

and the partition function after integration over fermionic degrees of freedom becomes 


$$
Z=\int \mathcal{D} x \operatorname{det}\left[i \partial_{t}+W\right] e^{-\int_{0}^{T} d t\left[\frac{1}{2} \dot{x}^{2}-V\right]}
$$

The fermionic determinant, as usual, is $\prod_{n} \lambda_{n}$ where $\lambda_{n}$ are the eigenvalues of the onedimensional Dirac operator $i \partial_{t}+W$, namely

$$
\left[i \partial_{t}+W\right] \varphi_{n}=\lambda_{n} \varphi_{n}
$$

Equation (5) can be formally integrated out and the exact solution is

$$
\varphi(t)=\varphi(0) e^{i \int_{0}^{t} d t^{\prime}\left(W\left(x\left(t^{\prime}\right)\right)-\lambda_{n}\right)}
$$

and using (3), one finds

$$
\int_{0}^{T} d t W-\lambda_{n} T=2 \pi\left(n+\frac{1}{2}\right)
$$

$\forall n \in \mathbf{Z}$.

On the other hand a quantization condition similar to the flux quantization condition found in [5] for the Thirring model can be derived directly from the equation of motion for the fermion variable $\psi$

$$
\left(i \partial_{t}+W\right) \psi=0
$$

with the boundary condition $\psi(T)=-\psi(0)$; one finds the condition

$$
\int_{0}^{T} d t W=2 \pi\left(m+\frac{1}{2}\right)
$$

However (7) and (9) are compatible if and only if $\lambda_{n}=0$ is one of the eigenvalues of the Dirac operator.

The flux quantization condition gives a restriction on the possible trayectories $x(t)$. The path integral (4) is decomposed in topological sectors and it is replaced by a sum (over $m$ ) of integrals of the trayectories satisfying (9). In general one can introduce a new parameter $\theta$ and an additional weight $e^{i m \theta}$ to each sector.

As a simple example that shows explicitly the physical meaning of the flux quantization condition, let us consider a linear function $W=\lambda x$. In this case (9) becomes 


$$
\int_{0}^{T} d t x(t)=\frac{2 \pi}{\lambda}\left(m+\frac{1}{2}\right)
$$

If one introduces the decomposition in modes

$$
x(t)=x^{(0)}+\sum_{n \neq 0} a_{n} \sin \left(\frac{n \pi}{T} t\right)
$$

then the flux quantization condition fixes the zero mode $x^{(0)}$ in terms of the integer $m$

$$
x^{(0)}=\frac{2 \pi}{\lambda T}\left(m+\frac{1}{2}\right) \text {, }
$$

and the sum over topological sectors replaces the integral over the zero mode in the original path integral (四).

For other choices of $W$ one has similar results, a relation (which will be more involved) between the zero mode $x^{(0)}$ and the integer $m$ but the physical meaning of the flux quantization condition (9) is the same.

\section{THIRRING MODEL}

In [5] a first discussion of the possible implications of a compactified light-like coordinate in the quantization of the Thirring model was presented. The study was based on the identification of a relation of the integration over the auxiliary variable (in the ligth-cone quantization) $\psi_{R}$ with a quantum mechanical determinant. Consistency of the determinant lead to a quantization of the product of the left-handed charge $Q_{L}$ and the Thirring coupling $g^{2}$ and then to the identification of many more excitations than those expected perturbatively.

In this section a complementary analysis of the quantization of the Thirring model compatible with the conservation of the vector current is presented. The starting point is the lagrangian,

$$
\mathcal{L}^{T h}=\psi_{L}^{\dagger}\left(i \partial_{+}+A_{+}\right) \psi_{L}+\psi_{R}^{\dagger}\left(i \partial_{-}+A_{-}\right) \psi_{R}+\frac{1}{2 g^{2}} A_{+} A_{-},
$$


which, eliminating the auxiliary vector field $A_{\mu}$, reproduces the lagrangian of the massless Thirring model in ligth cone coordinates $x^{ \pm}=t \pm x$. In order to identify the consequences of the boundary conditions on the fermionic field at $x^{-}= \pm L$ we consider the solution of the equation $\left(i \partial_{-}+A_{-}\right) \psi_{R}=0$ for the rigth-handed fermionic field

$$
\psi_{R}\left(x^{+}, x^{-}\right)=e^{i \int_{0}^{x^{-}} d y^{-} A_{-}\left(x^{+}, y^{-}\right)} \psi_{R}\left(x^{+}, 0\right) .
$$

The most general boundary conditions compatible with charge conjugation symmetry are either periodic boundary conditions (p.b.c.) $\psi\left(x^{+}, L\right)=\psi\left(x^{+},-L\right)$ or antiperiodic boundary conditions (a.b.c) $\psi\left(x^{+}, L\right)=-\psi\left(x^{+},-L\right)$. In order to have a solution for $\psi_{R}$ compatible with the boundary conditions, the auxiliary field component $A_{-}$has to satisfy the restriction

$$
\begin{aligned}
& \int_{-L}^{L} d x^{-} A_{-}\left(x^{+}, x^{-}\right)=2 \pi N \text { for }(\text { p.b.c. }) \\
& \int_{-L}^{L} d x^{-} A_{-}\left(x^{+}, x^{-}\right)=2 \pi\left(N+\frac{1}{2}\right) \text { for (a.b.c.) }
\end{aligned}
$$

On the other hand if one eliminates the auxiliary field component $A_{+}$, one finds $A_{-}=$ $-2 g^{2} \psi_{L}^{\dagger} \psi_{L}$ and the restriction on the auxiliary field component $A_{-}$can be translated into a quantization condition on the left-handed chiral charge $Q_{L}=\int_{-L}^{L} d x^{-} \psi_{L}^{\dagger} \psi_{L}$,

$$
\begin{aligned}
Q_{L} & =\frac{\pi}{g^{2}} N \text { for }(\text { p.b.c. }) \\
Q_{L} & =\frac{\pi}{g^{2}}\left(N+\frac{1}{2}\right) \text { for (a.b.c.) }
\end{aligned}
$$

giving an important information on the non-perturbative spectrum of the model [5].

One can also see how the standard analysis of the model based on bosonization is modified due to the presence of a compact light-like dimension. In this case one has to consider the effective fermionic action, $\Gamma_{f}[A]$, defined by

$$
e^{i \Gamma_{f}\left[A_{+}, A_{-}\right]}=\int d \psi^{\dagger} d \psi e^{i \int d^{2} x\left(\psi_{L}^{\dagger}\left(i \partial_{+}+A_{+}\right) \psi_{L}+\psi_{R}^{\dagger}\left(i \partial_{-}+A_{-}\right) \psi_{R}\right)},
$$

together with the two-dimensional representation of the (auxiliary) vector field in terms of the derivatives of two scalar fields. Using ligth-cone coordinates, with $-L \leq x^{-} \leq L$, one has $A_{+}=\partial_{+} \chi, A_{-}=\partial_{-} \phi$ with 


$$
\begin{aligned}
& \chi\left(x^{+}, x^{-}\right)=\sum_{n} \chi_{n}\left(x^{+}\right) e^{\frac{i n \pi}{L} x^{-}}, \\
& \phi\left(x^{+}, x^{-}\right)=\sum_{n \neq 0} \phi_{n}\left(x^{+}\right) e^{\frac{i n \pi}{L} x^{-}}+\frac{A_{-}^{(0)}\left(x^{+}\right)}{2 L} x^{-} .
\end{aligned}
$$

We have made explicit in the expansion the zero mode $A_{-}^{(0)}$,

$$
A_{-}^{(0)}=\int_{-L}^{L} d x^{-} A_{-}
$$

which instead of being a function of $x^{+}$becomes a discrete variable as a consequence of the restriction (15) due to the boundary conditions.

When the result for the fermionic effective action

$$
\Gamma_{f}\left[A_{+}, A_{-}\right]=\frac{1}{2 \pi} \int d^{2} x\left(A_{+} A_{-}-\frac{1}{2} A_{+} \frac{\partial_{-}}{\partial_{+}} A_{+}-\frac{1}{2} A_{-} \frac{\partial_{+}}{\partial_{-}} A_{-}\right),
$$

where the regularization arbitrariness has been fixed by conservation of the vector current, is combined with the $x^{-}$-expansion of $A_{ \pm}$one finds

$$
\Gamma_{f}\left[\partial_{+} \chi, \partial_{-} \phi\right]=\frac{A_{-}^{(0)}}{2 \pi} \Delta \chi_{0}+\sum_{n} \frac{i n}{4 L} \int d x^{+}\left(\chi_{-n}-\phi_{-n}\right) \partial_{+}\left(\chi_{n}-\phi_{n}\right)
$$

where $\Delta \chi_{0}=\int d x^{+} \partial_{+} \chi_{0}=\chi_{0}(\infty)-\chi_{0}(-\infty)$.

The bosonized action $\mathcal{S}_{\text {bos }}^{T h}$ is obtained by adding to (21) the term quadratic in the auxiliary field of the original lagrangian (13),

$$
\begin{aligned}
\mathcal{S}_{\text {bos }}^{T h} & =\Gamma_{f}\left[\partial_{+} \chi, \partial_{-} \phi\right]+\frac{1}{2 g^{2}} \int d^{2} x \partial_{+} \chi \partial_{-} \phi \\
& =\left(1+\frac{\pi}{g^{2}}\right)\left[\frac{A_{-}^{(0)}}{2 \pi} \Delta \chi_{0}-\sum_{n}\left(\frac{i n}{2 L}\right) \int d x^{+} \phi_{-n} \partial_{+} \chi_{n}\right. \\
& \left.\times \sum_{n}\left(\frac{i n}{4 L}\right) \int d x^{+}\left(\chi_{-n} \partial_{+} \chi_{n}+\phi_{-n} \partial_{+} \phi_{n}\right)\right] .
\end{aligned}
$$

The bosonized action is a sum of terms (non-zero modes) quadratic in the derivatives of two scalar fields in a two dimensional space with $-L \leq x^{-} \leq L$ plus a contribution of the zero modes which involves the discrete variable $A_{-}^{(0)}$ and $\Delta \chi_{0}$ exclusively. All the implications of the compactification of the light-like coordinate $x^{-}$are contained in the contribution of the zero modes. 
The first restriction on these zero modes comes from the relation

$$
\begin{aligned}
& \left(\frac{-g^{2}}{\pi}\right) n_{L}=N \text { for (p.b.c.) } \\
& \left(\frac{-g^{2}}{\pi}\right) n_{L}=\left(N+\frac{1}{2}\right) \text { for (a.b.c.) }
\end{aligned}
$$

which is the quantization condition (16) with $Q_{L}$ replaced by the number $n_{L}$ of left-handed fermions at fixed $x^{+}$.

In the case of antiperiodic boundary conditions $g^{2} / \pi$ has to be a rational number with even denominator, $g^{2} / \pi=p / 2 q$ and the number of left-handed fermions has to be a multiple of $q, n_{L}=q \hat{n}_{L}$. The integer $N$ which fixes the value of the discrete variable $A_{-}^{(0)}$ (see (15)) is given by

$$
N=-\left(\frac{p \hat{n}_{L}+1}{2}\right)
$$

If one introduces an aditional weight factor $e^{i \theta N}$ to each sector, the sum over $N$ can be easily evaluated using the only term which depends on the zero modes, $e^{i_{-}^{A_{(0)}}} \frac{\chi_{0}}{2 \pi}$; one finds a quantization condition on $\Delta \chi_{0}$

$$
\frac{p}{2}\left[\left(1+\frac{\pi}{g^{2}}\right) \Delta \chi_{0}+\theta\right]=2 \pi M,
$$

with integer M.

Taking into account that $A_{+}=-2 g^{2} \psi_{R}^{\dagger} \psi_{R}$ and the dependence of $\psi_{R}$ on $x^{-}$given by the solution (14) for the right-handed field, one has an auxiliary field component $A_{+}$which does not depend on $x^{-}$. This means that one can take $\chi_{n}=0$ in the mode expansion and also that $\Delta \chi_{0}$ can be reexpressed in terms of the original fermionic variables:

$$
\Delta \chi_{0}=\frac{1}{2 L} \int_{-L}^{L} d x^{-} \int d x^{+} A_{+}=-2 g^{2} \int d x^{+} \psi_{R}^{\dagger} \psi_{R}
$$

Then the quantization condition for $\Delta \chi_{0}$ (25) can be written in the form

$$
\frac{p}{2}\left[\frac{\theta}{2 \pi}-\left(1+\frac{p}{2 q}\right) n_{R}\right]=M
$$


where the integer $n_{R}$ is the number of right-handed fermions for fixed $x^{-}$. From this relation one concludes that $\theta / 2 \pi$ has to be also a rational number, $\frac{\theta}{2 \pi}=\frac{m}{2 p q}$ and the number of righthanded fermions is restricted by the condition that $m-p(p+2 q) n_{R}$ has to be a multiple of $4 q$.

In the case of periodic boundary conditions one has a trivial solution to the quantization condition for the zero modes with $n_{L}=0$. In this case $N=0$ and there is no possibility to introduce an angle $\theta, g^{2} / \pi$ can be any real number and the zero modes do not appear in the action. Together with this trivial case there is another solution to the quantization condition for the zero modes which requires $g^{2} / \pi$ to be a rational number as in the case of antiperiodic boundary conditions, $g^{2} / \pi=p / q$. In this case one also finds that the number of left-handed fermions is a multiple of $q, n_{L}=q \hat{n}_{L}$ and the integer $N$ is in this case $N=-p \hat{n}_{L}$. The quantization condition for $\Delta \chi_{0}$ which results from the sum over $N$ with an aditional weigth factor $e^{i \theta N}$ is

$$
p\left[\frac{\theta}{2 \pi}-\left(1+\frac{p}{q}\right) n_{R}\right]=M .
$$

In this case one finds that $\theta / 2 \pi$ has to be a rational number, $\frac{\theta}{2 \pi}=\frac{m}{p q}$ and the number of right-handed fermions has to be such that $m-p^{2} n_{R}$ is a multiple of $q$.

We end up this section emphasizing that the light-cone quantization provides new nonperturbative information that in the usual spacetime quantization we cannot see. In particular, the existence of new excitations, the zero modes and the preponderant role played by the boundary conditions are new ingredients that are absent in the spacetime quantization

\section{SCHWINGER MODEL}

The analysis in the previous section of the implications on the quantization of a compactified light-like coordinate for the Thirring model can be easily applied to the Schwinger model. The discussion of boundary conditions based on the solution (14) for $\psi_{R}$ and the expansion of the fermionic effective action (21) can be directly applied with the replacement of the auxiliary vector field of the Thirring model by the dynamical gauge field in the 
Schwinger model. Then, instead of the quadratic term in the auxiliary vector field of the Thirring model, one has the action of the gauge field

$$
\mathcal{S}_{g}=\frac{1}{4 e^{2}} \int d^{2} x\left(\partial_{+} A_{-}-\partial_{-} A_{+}\right)^{2}
$$

Using the $x^{-}$-expansion of the gauge field one has

$$
\partial_{+} A_{-}-\partial_{-} A_{+}=-\sum_{n}\left(\frac{i n \pi}{L}\right) \partial_{+}\left(\chi_{n}-\phi_{n}\right) e^{\frac{i n \pi}{L} x^{-}}
$$

The sum of the fermionic effective action (21) and the gauge field action (29) leads to the bosonized action of the Schwinger model

$$
\begin{aligned}
\mathcal{S}_{\text {bos }}^{S M}= & \frac{A_{-}^{(0)}}{2 \pi} \Delta \chi_{0}+\frac{1}{4 \pi} \sum_{n}\left(\frac{i n \pi}{L}\right) \int d x^{+}\left(\chi_{-n}-\phi_{-n}\right) \partial_{+}\left(\chi_{n}-\phi_{n}\right)- \\
& \frac{1}{4 e^{2}} \sum_{n}\left(\frac{i n \pi}{L}\right)^{2} \int d x^{+} \partial_{+}\left(\chi_{-n}-\phi_{-n}\right) \partial_{+}\left(\chi_{n}-\phi_{n}\right) .
\end{aligned}
$$

Together with the zero mode contribution already found in the Thirring model, we have an action for the combination $\chi-\phi$ of the two scalar fields which appear in the twodimensional decomposition of the gauge field. The fact that only one combination of the two scalars fields appear in the action is a consequence of gauge invariance. From the relative coefficient of the two terms involving $\chi_{n}-\phi_{n}$ one reproduces the well known mass gap of the model $\left(m^{2}=e^{2} / \pi\right)$.

It is also clear that introducing an external source $J_{\mu}$ one has

$$
\partial_{\mu}<\bar{\psi} \gamma^{\mu} \psi>=\left.\left[\partial_{-} i \frac{\delta}{\delta J_{-}}+\partial_{+} i \frac{\delta}{\delta J_{+}}\right] e^{i \Gamma_{f}[A+J]}\right|_{J=0}=0
$$

and

$$
\partial_{\mu}<\bar{\psi} \gamma^{\mu} i \gamma_{5} \psi>=\left.\left[\partial_{-} i \frac{\delta}{\delta J_{-}}-\partial_{+} i \frac{\delta}{\delta J_{+}}\right] e^{i \Gamma_{f}[A+J]}\right|_{J=0}=\frac{e}{\pi}\left(\partial_{+} A_{-}-\partial_{-} A_{+}\right),
$$

which reproduces the conservation of the vector current and the anomaly in the axial current.

In the Schwinger model there is no analog of the relation found in the Thirring model between the (auxiliary) vector field and the fermionic variables. Then the discussion of the zero modes is very simple. There is no restriction on the integer $N$ which appears in the 
quantization condition (15) on the zero mode of the gauge field component $A_{-}$. On the other hand if one sums over all posible values of $N$ with an additional relative weight $e^{i \theta N}$ then one has a simple quantization condition for $\Delta \chi_{0}$

$$
\Delta \chi_{0}+\theta=2 \pi M
$$

It is remarkable that, although one has

$$
\int d^{2} x F_{01}=-\sum_{n}\left(\frac{i n \pi}{L}\right) \int d x^{+} \partial_{+}\left(\chi_{n}-\phi_{n}\right) \int d x^{-} e^{\frac{i n \pi}{L} x^{-}}=0
$$

and then apparently a trivial vacuum structure, the two integer structure of the Schwinger model [7] is easily derived from the contribution of the zero modes ( $N$ corresponding to the zero mode of the $A_{-}$component and $M$ corresponding to the zero mode of $A_{+}$).

The analysis of the implications of a light-like compact coordinate in the quantization of a theory with a dynamical vector field can also be applied to the chiral Schwinger model. There are two different models

$$
\begin{aligned}
& \mathcal{L}_{L}^{C S M}=\psi_{L}^{\dagger}\left(i \partial_{+}-A_{+}\right) \psi_{L}+\psi_{R}^{\dagger} i \partial_{-} \psi_{R}-\frac{1}{4} F_{\mu \nu} F^{\mu \nu} \\
& \mathcal{L}_{R}^{C S M}=\psi_{L}^{\dagger} i \partial_{+} \psi_{L}+\psi_{R}^{\dagger}\left(i \partial_{-}-A_{-}\right) \psi_{R}-\frac{1}{4} F_{\mu \nu} F^{\mu \nu}
\end{aligned}
$$

In the first case the equation for the right-handed field does not involve the vector field and there is no quantization condition on the zero modes of the vector field. The effective fermionic action is given in this case by

$$
\Gamma_{f}^{L}\left[A_{+}, A_{-}\right]=\frac{1}{2 \pi} \int d^{2} x\left(a A_{+} A_{-}-\frac{1}{2} A_{+} \frac{\partial_{-}}{\partial_{+}} A_{+}\right),
$$

where, as a consequence of the chiral coupling, the non-local contribution involves only the $A_{+}$component and the local term has a coeficient with a regularization dependent parameter $a$ due to the lack of gauge invariance [8]. The bosonized action, obtained by using the decomposition of the vector field in terms of two scalar fields (18), is

$$
\begin{gathered}
\mathcal{S}_{\text {bos }}^{L C S M}=\frac{a}{2 \pi} \int d x^{+} A_{-}^{(0)} \chi_{0}-\frac{a}{2 \pi} \sum_{n}\left(\frac{i n \pi}{L}\right) \int d x^{+} \phi_{-n} \partial_{+} \chi_{n}+ \\
\frac{1}{4 \pi} \sum_{n}\left(\frac{i n \pi}{L}\right) \int d x^{+} \chi_{-n} \partial_{+} \chi_{n}-\frac{1}{4 e^{2}} \sum_{n}\left(\frac{i n \pi}{L}\right)^{2} \int d x^{+} \partial_{+}\left(\chi_{-n}-\phi_{-n}\right) \partial_{+}\left(\chi_{n}-\phi_{n}\right) .
\end{gathered}
$$


Note that in this case there is no quantization condition on the zero mode of $A_{-}$and $\theta$-vacua are absent, a result derived recently [9] in the light-cone hamiltonian formulation.

In the chiral model with an interacting right-handed component one has the same quantization condition (15) on the zero mode of $A_{-}$as in the Schwinger model and the bosonized action is

$$
\begin{gathered}
\mathcal{S}_{\text {bos }}^{R C S M}=\frac{a}{2 \pi} A_{-}^{(0)} \Delta \chi_{0}-\frac{a}{2 \pi} \sum_{n}\left(\frac{i n \pi}{L}\right) \int d x^{+} \phi_{-n} \partial_{+} \chi_{n}+ \\
\frac{1}{4 \pi} \sum_{n}\left(\frac{i n \pi}{L}\right) \int d x^{+} \phi_{-n} \partial_{+} \phi_{n}-\frac{1}{4 e^{2}} \sum_{n}\left(\frac{i n \pi}{L}\right)^{2} \int d x^{+} \partial_{+}\left(\chi_{-n}-\phi_{-n}\right) \partial_{+}\left(\chi_{n}-\phi_{n}\right) .
\end{gathered}
$$

The treatment of the zero modes is similar to the gauge invariant case with the quantization condition for $\Delta \chi_{0}(34)$ replaced by

$$
a \Delta \chi_{0}+\theta=2 \pi M
$$

\section{DISCUSSION}

We end up by considering the possibility to extend the analysis of the Thirring model, Schwinger model and chiral Schwinger models presented in this work. One property of all these models crucial in all the discussion is the absence of a mass term for the fermion allowing to decouple the two chiral components in the equation for the fermion field. With this restriction there are two possible extensions to consider.

The first generalization is to consider a non-abelian generalization where one has several fermionic fields instead of a single Dirac field. One will have an equation for the righthanded fermion field multiplet with the vector field $A$ in the abelian cases replaced by a Lie algebra valued vector field and also a generalization of the boundary condition for the rigth-handed field $\psi_{R}\left(x^{+}, L\right)=e^{2 \pi i \Gamma} \psi_{R}\left(x^{+},-L\right)$ with a matrix $\Gamma$ in internal space. One will have in this case a non-abelian generalization of the quantization condition (15),

$$
\mathcal{P}_{x^{-}} e^{i \int_{-L}^{L} d x^{-} A_{-}}=e^{2 \pi i \Gamma}
$$


where $\mathcal{P}_{x^{-}}$denotes a path ordering on the light-like coordinate $x^{-}$. The study of the consequences of this quantization condition in a non-abelian generalization of the Thirring model as well as in models with dynamical vector fields deserves further investigation.

A second generalization of the present work is to try to go beyond two space-time dimensions. In higher dimensions the solution of the right-handed fermion field equation can be expressed also as a path-ordered exponential

$$
\psi_{R}\left(x^{+}, x^{-}, \vec{x}_{\perp}\right)=\mathcal{P}_{x^{-}} e^{i \int_{0}^{x^{-}} d y^{-}\left[A_{-}\left(x^{+}, y^{-}, \vec{x}_{\perp}\right)-i \vec{\alpha}_{\perp}\left(\vec{\partial}_{\perp}+i \vec{A}_{\perp}\left(x^{+}, y^{-}, \vec{x}_{\perp}\right)\right)\right.} \psi_{R}\left(x^{+}, 0, \vec{x}_{\perp}\right) .
$$

Then the choice of periodic or antiperiodic boundary conditions for the right-handed fermionic field in light-like coordinate $x^{-}$leads to a higher dimensional quantization condition

$$
\mathcal{P}_{x^{-}} e^{i \int_{-L}^{L} d y^{-}\left[A_{-}\left(x^{+}, y^{-}, \vec{x}_{\perp}\right)-i \vec{\alpha}_{\perp}\left(\vec{\partial}_{\perp}+i \vec{A}_{\perp}\left(x^{+}, y^{-}, \vec{x}_{\perp}\right)\right)\right]}= \pm 1
$$

which is more difficult to analyze than the two dimensional case due to the presence of the additional transverse coordinates $\vec{x}_{\perp}$. This should be the starting point for a study of the consequences of a compactified light-like coordinate $x^{-}$on the quantization of a theory with a massless fermionic field.

\section{Acknowledgements}

We would like to thank J. Zanelli by several interesting discussions about this paper. This work was partially supported by CICYT (Spain) project AEN-97-1680 and grants 1980788, 7980045 from FONDECYT-Chile, and DICYT-USACH. 


\section{REFERENCES}

[1] S. Brodsky, H.-C. Pauli and S. Pinsky, hep-ph/9705477, Phys. Rep. 301, 299 (1998) and references therein.

[2] M. M. Brisudova, R. Perry and K.G. Wilson, hep-ph/9607280, Phys. Rev. Lett. 78, 1227 (1997); S. D. Glazek and K. G. Wilson, hep-th/9707028, Phys. Rev. D57, 3558 (1998).

[3] D. Bigatti and L. Susskind, hep-th/9711063, Phys. Lett. B425, 351 (1998).

[4] T. Maskawa and K. Yamawaki, Prog. Theor. Phys. 56270 (1976); S. Brodsky and H. -C. Pauli, Phys. Rev.32, 1993 (1985).

[5] J.L. Cortés, J. Gamboa, I. Schmidt and J. Zanelli, hep-th/9809051, Phys. Lett. B444, 451 (1998).

[6] E. Gildener and A. Patrascioiu, Phys. Rev. D16, 1802 (1977).

[7] E. Abdalla, M.B. Abdalla and K.D. Rothe, Non-perturbative methods in 2 dimensional quantum field theory pg. 301, (World Scientific, Singapore, 1991)

[8] J. Jackiw and R. Rajamaran, Phys. Rev. Lett. 54, 1219 (1985).

[9] P.P. Srivastava, hep-th/9811225, Phys. Lett.B (in press). 